\title{
Review
}

\section{Pheochromocytoma in Pregnancy: A Review of the Japanese Literature}

\author{
SeIICHI OISHI AND Tatsuo SATO \\ The Third Department of Internal Medicine, Kumamoto \\ University School of Medicine, Kumamoto 860, Japan
}

\section{Introduction}

PHEOCHROMOCYTOMA in association with pregnancy is rare, but very important because of the hazardous complications and poor prognosis for both mother and fetus [1-5]. A diagnosis of pheochromocytoma during pregnancy was difficult and was rarely made prior to delivery. The remarkable similarity between the clinical manifestations of pheochromocytoma and toxemia of pregnancy or eclampsia led to the pitfall mentioned above [6, 7]. However, mortality in both mother and fetus could be lowered when the diagnosis was made antepartum $[2,7,8]$. For example, maternal mortality before 1969 was $48 \%$, and it fell to $26 \%$ in the period 1969-1979 [9] and continued to fall to $17 \%$ in $1980-1989$ [2]. In this paper, we review the 35 cases with pheochromocytoma in pregnancy in the Japanese literature, including our 3 cases $[10,11]$, and demonstrate diagnostic criteria including diagnostic imaging procedures that enable an early, correct diagnosis to be made.

\section{Materials and Methods}

Thirty-five patients with pheochromocytoma in pregnancy including our 3 patients reported in the Japanese literature from 1958 to 1993 have been reviewed. Details of these 35 patients are summarized in Table 1.

Received: July 14, 1993

Accepted: January 21, 1994

Correspondence to: Dr. Seiichi OISHI, The Third Department of Internal Medicine, Kumamoto University School of Medicine, 1-1-1 Honjo, Kumamoto 860, Japan

\section{Results}

\section{Clinical characteristics}

The 35 patients had a total of 57 pregnancies while the tumor was present. For eighteen patients $(51 \%)$ it was the first pregnancy, for 11 patients (31\%) the second or a subsequent pregnancy, and for 6 patients $(17 \%)$ the number of pregnancies was not known. The mean age of the 35 patients was $28.8 \pm 5.0$ (mean $\pm S D$ ) years. The youngest patient was 21 years of age and the oldest was 44 . Twentyfour patients $(69 \%)$ were diagnosed in the third decades, 9 patients (26\%) in the fourth decade, and 2 patients $(6 \%)$ in the fifth decade.

\section{Clinical manifestations (symptoms and signs)}

The clinical symptoms, such as headache, palpitation, sweating, and hypertension, which related to pheochromocytoma and suggested excess circulating catecholamines, generally appeared in the third trimester. The incidence in the three trimesters of pregnancy was as follows: first, 4 patients (11\%); second, 7 patients (20\%); third, 23 patients (66\%) (Fig. 1). Paroxysmal hypertension was found in $27(77 \%)$ of the 35 patients, and sustained hypertension in 8 patients (23\%). However, 3 of 8 patients initially had paroxysmal hypertension, and later had sustained hypertension.

\section{Differential diagnosis}

An initial diagnosis of toxemia of pregnancy was made in 17 patients $(49 \%)$ of the 35 pregnancies, pregnancy nephritis in $2(6 \%)$, chronic glomerulonephritis in $1(3 \%)$, essential hypertension in $3(9 \%)$, cerebral thrombosis in $1(3 \%)$, heart 


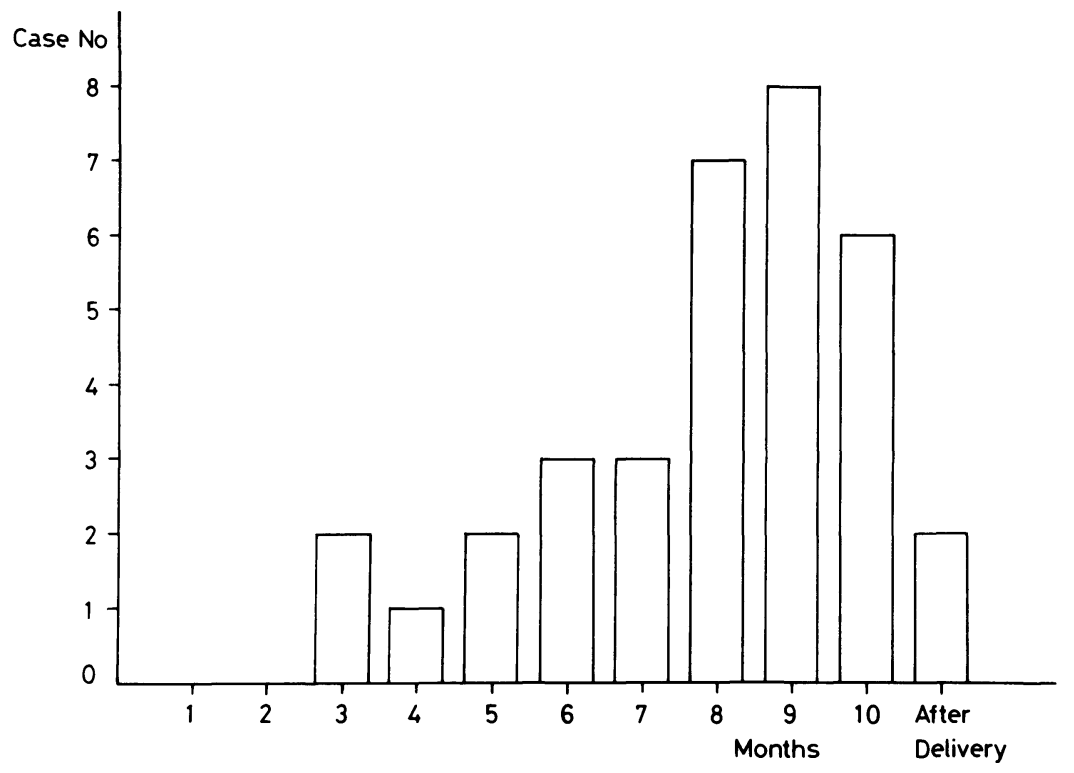

Fig. 1. Time of onset of symptoms and months of pregnancy.

failure in $1(3 \%)$, and pheochromocytoma in 10 (29\%). A diagnosis of pheochromocytoma was made before delivery in 11 patients $(31 \%)$, after delivery in 22 patients $(63 \%)$, at autopsy in one patient $(3 \%)$, and unknown in one patient (3\%). After 1981, a diagnosis of pheochromocytoma was made before delivery in 5 patients (29\%), after delivery in 10 patients $(59 \%)$, at autopsy in one patient $(6 \%)$ and unknown in one patient $(6 \%)$ of 17 patients.

\section{Diagnosis}

The biochemical diagnosis was confirmed by measuring urinary catecholamines. The mean urinary noradrenaline and adrenaline excretions were $1618 \pm 1380 \mu \mathrm{g} / 24 \mathrm{~h}$ and $131 \pm 165 \mu \mathrm{g} / 24 \mathrm{~h}$, which were significantly higher than normal values (22 \pm $3.0 \mu \mathrm{g} / 24 \mathrm{~h}$ and $5.0 \pm 2.1 \mu \mathrm{g} / 24 \mathrm{~h}, \mathrm{n}=250$ ).

Diagnostic imaging procedures have been greatly improved, especially in the past 10 years. The diagnosis of pheochromocytoma was confirmed histologically in each of the 35 patients either at surgery or autopsy.

\section{Localization of pheochromocytoma}

The tumor was located in the right adrenal in 16 patients $(46 \%)$, in the left in $12(34 \%)$, there were bilateral adrenal tumors in $4(11 \%)$, in the extraadrenal in $2(6 \%)$, and of unknown origin in 1 patient (3\%). The mean tumor weight was $77.4 \pm$ $66.9 \mathrm{~g}(\mathrm{n}=30)$ except for 2 large ones (900 $\mathrm{g}$ and $2770 \mathrm{~g}$ ). The mean tumor weight in the first pregnancy was $71.0 \pm 61.4 \mathrm{~g}(\mathrm{n}=14)$, and that in the second or later pregnancies was $67.2 \pm 68.9 \mathrm{~g}$ $(n=11)$, and the differences between them were not significant.

\section{Delivery methods}

The methods of delivery were as follows: artificial abortion in 3 patients (9\%), artificial immature labor in 5 patients (14\%), normal labor in 8 patients (23\%), caesarean section in 14 patients $(40 \%)$, one died (3\%), and the method was unknown in 4 patients (11\%). After 1981 caesarean section was commonly performed in $11(65 \%)$ of the 15 patients, and after 1986 it was performed in all cases.

\section{Outcome}

The total maternal mortality rate was $11 \%$ (4 patients). After 1981, it was reduced to $6 \%$ (1 patient). The maternal mortality rate was 0 out of 17 cases in the first pregnancy and it was 2 out of 11 in the second or more pregnancies. The diagnosis of pheochromocytoma was made at autopsy in all of the four patients who died. And none of them had been suspected of the association with pheochromocytoma. One mother worsened with labor 
and died of heart failure during the antenatal period. Two mothers died within $72 \mathrm{~h}$ after delivery, and one died about 9 days after delivery. Twentyeight patients were cured after the removal of the tumor. Fetal death occurred in $7(22 \%)$ of 32 patients recorded. After 1981, it was reduced to one patient $(6 \%)$.

\section{Discussion}

Here we have reviewed the 35 cases of pheochromocytoma associated with pregnancy reported in the Japanese literature from 1958 to 1993. Pheochromocytoma produces similar symptoms and signs in both pregnant and nonpregnant patients, but the diagnosis may be overlooked in pregnancy because of the rarity of the association and because the clinical picture often mimics that of pre-eclampsia [6, 12]. An unrecognized pheochromocytoma is particularly dangerous [4, 13], as potentially fatal hypertensive crises may be precipitated by anesthesia, vaginal delivery, the mechanical effects of the gravid uterus, uterine contractions, and even vigorous fetal movements.

The maternal mortality rate was improved during this study. In 35 patients with pheochromocytoma in pregnancy, although the maternal mortality rate before 1979 was $17 \%$, it fell to $6 \%$ in the period 1980-1993. When the diagnosis was made antepartum, the maternal mortality fell to zero, but it was made in only $32 \%$ of the patients.

Fetal growth retardation may occur secondary to reduced uteroplacental circulation, and fetal hypoxia and death may occur during acute hypertensive crises, maternal collapse or death [14]. Antepartum diagnosis and alpha-blockade reduce the fetal mortality rate [15].

Accordingly, antenatal recognition of a pheochromocytoma is most important because of the improved maternal and fetal outcome [2]. However, pheochromocytoma was diagnosed antenatally in only $11(32 \%)$ of the 34 patients in the present Japanese review (Table 1) compared with $25 \%$ before 1969 and $52 \%$ in the period 1969 1979 [9] and 53\% in the period 1980-1987 [2] in the world literature.

\section{Clinical manifestations (symptoms and signs)}

The symptoms most suggestive of pheochro- mocytoma were paroxysmal hypertension in both our study and other reports [1]. Most of these patients had fluctuating, often severe, hypertension associated with a variety of symptoms and signs, the commonest being headache, excessive perspiration, palpitation and tachycardia. The clinical symptoms generally appeared in the third trimester [1]. This may be due to the mechanical effects of the gravid uterus, uterine contractions, and vigorous fetal movements. The intraabdominal pressure changes accompanying pregnancy may account for the increased release of catecholamines from a pheochromocytoma which may previously have been relatively quiescent. Labor may further compress a pheochromocytoma and increase the release of catecholamines from the tumor.

\section{Differential diagnosis}

The initial diagnosis was toxemia of pregnancy which appeared in 16 patients ( $47 \%$ of the 34 pregnancies) and pheochromocytoma in 10 (29\%). Hume [12] pointed out that pregnancy complicated by pheochromocytoma may be confused with (a) toxemia, when sustained hypertension is present during the early stages of pregnancy, (b) preeclampsia, when headache, edema, tachycardia, and hypertension are seen in late pregnancy, or (c) a ruptured uterus and shock during or immediately after labor. He said that patients with toxemia of pregnancy, preeclampsia, or unexplained attacks should be screened for the presence of pheochromocytoma. The most important aid to early diagnosis is to think of pheochromocytoma. Hypertension is very common in pregnancy, and patients must be selected for investigation. Pheochromocytoma should be excluded in all pregnant women with severe or intermittent hypertension, with hypertension associated with the paroxysmal symptoms.

\section{Diagnosis}

\section{1) Biochemical tests}

Estimation of urinary catecholamines is essential in a properly collected $24 \mathrm{~h}$ urine sample, preferably obtained during or immediately following a hypertension episode. Pharmacological tests such as the Regitine test and Glucagon test are no longer necessary and have been associated with maternal and fetal death. 
2) Preoperative localization of pheochromocytoma

Diagnostic imaging procedures have greatly improved, especially in the past 10 years, and diagnostic imaging procedures that do not employ ionizing radiation are obviously ideal for use in pregnant patients. Abdominal ultrasound study is safe and easy to use in displaying the tumor. The use of computed tomography (CT) scan in pregnant women should be restricted because of irradiation of the fetus. However, magnetic resonance (MR) imaging has been used recently during pregnancy $[17,18]$. The use of MR imaging permitted early localization of a retrouterine tumor when it would have been undesirable for the woman to undergo a CT scan because of radiation exposure. It produced high quality images and had the advantage that it did not employ ionizing radiation. Therefore, MR imaging is thought to be most useful in detecting and localizing pheochromocytoma during pregnancy.

3) Site of pheochromocytoma

Ninety percent of pheochromocytomas arise in the adrenal medulla and $98 \%$ occur within the abdomen [16]. Bilateral adrenal tumors occur in $10 \%$ of cases. In the present study, the tumor site was the right adrenal in $16(46 \%)$, left adrenal in 12 $(34 \%)$, bilateral adrenal in $4(11 \%)$ and intra-abdominal extra-adrenal in $2(6 \%)$. One of these last 2 patients had two multiple extra-adrenal tumors (Table 1). The tumor site was unknown in one patient.

\section{Treatment}

1) Preoperative evaluation

Treatment with an alpha-adrenergic blocking agent to control hypertension before surgery should be started as soon as the diagnosis of pheochromocytoma has been established, or sooner if pheochromocytoma is strongly suspected [2, 19]. Prazosin (a post-synaptic alphaadrenoceptor blocking agent) [8] and labetalol (an alpha and beta-adrenoceptor blocking agent) are the choice of drugs during pregnancy because they have no adverse effects on the fetus. Beta-blockade should not be used without prior alpha-blockade and might lead to generalized vasoconstriction and a rise in the blood pressure. After adequate alphablockade has been established, beta-blockers may be used to control tachycardia and arrhythmias.

\section{2) Operative management}

The definitive treatment of pheochromocytoma is surgical removal [20]. When the diagnosis is made before 23 weeks gestation, the tumor should be removed once adrenergic blockade has been achieved. Although up until 1979 only one fetus survived out of the 5 pregnancies in which surgical intervention was undertaken before 24 weeks gestation [9], there has been a successful fetal outcome in 7 of the 8 more recently reported cases [2]. After 24 weeks gestation uterine size makes abdominal exploration and access to the tumor difficult unless the woman is first delivered. Optimum results are obtained if surgery is delayed until fetal maturity is reached. Then, under adrenergic blockade, elective caesarean section should be performed, followed immediately by exploration for the tumor [15].

3) Delivery methods and outcome (maternal mortality and effect on the fetus)

In the past, vaginal delivery was carried out and resulted in a higher maternal mortality $(31 \%)$ than caesarean section (19\%) [9]. Of the 11 women in the present review, where a pheochromocytoma was diagnosed but not removed antenatally, delivery was by caesarean section in 5 , with concurrent resection of the tumor in 2, removal at a later date in 3; there were two neonatal deaths and no maternal death. Where pheochromocytoma was unrecognized before delivery, acute hypertensive crises or shock occurred in several patients during or shortly after both caesarean section and vaginal delivery, causing maternal death after caesarean section in one of 8 patients and following vaginal delivery in one of 7 . One maternal death occurred after caesarean section and postpartum confirmation of pheochromocytoma but before surgical treatment. Three other patients with unrecognized pheochromocytoma died during the antenatal period or after delivery. The diagnosis of pheochromocytoma was made at autopsy in all of the four patients in our study who died. In this study, after 1981, caesarean section was commonly performed in 11 of the 17 patients, and after 1986, caesarean section was performed in all cases. And also after 1981 , both maternal and fetal mortality were significantly reduced to one $(6 \%)$ of 17 patients. 


\section{Conclusions}

Pheochromocytoma is a rare disease but an important cause of hypertension and cardiovascular collapse in pregnancy. Although the maternal and fetal outcomes have improved, an unsuspected pheochromocytoma may still have disastrous consequences [4]. A high index of clinical suspicion must be maintained to achieve an early diagnosis. All pregnant women with hypertension $[6,12]$ associated with any unusual features, particularly those with severe or symptomatic hypertension, headache, excessive sweating or palpitations, or with a family history of pheochromocytoma, should have their $24 \mathrm{~h}$ urinary catecholamine output checked. Then abdominal ultrasound and/or MR imaging offer the best methods of tumor localization. Adequate alpha-blockade is essential and beta-blockade may also be required. Tumor resection before 28 weeks gestation has been shown to have a good fetal outcome. In later pregnancy, delivery by caesarean section when fatal maturity is reached, followed by tumor resection, either at the time of casearean section or later, is the best. We conclude that early diagnosis is the key to a better prognosis in pheochromocytoma associated with pregnancy.

\section{Summary}

We review the 35 cases with pheochromocytoma associated with pregnancy including our own 3 cases in the Japanese literature from 1958 to 1993. In a total of 35 cases, the overall maternal mortality rate was $11 \%$ and the fetal loss $22 \%$. Recently, af- ter 1981, the maternal mortality rate and the fetal loss were significantly reduced to one patient $(6 \%)$. Although antepartum diagnosis of pheochromocytoma was made in only $32 \%$ of the patients, maternal mortality was reduced to zero and fetal loss to $18 \%$. An initial diagnosis of toxemia of pregnancy was made in 17 patients (49\%) and of pheochromocytoma in 10 patients $(29 \%)$. A diagnosis of pheochromocytoma was made before delivery in 11 patients $(31 \%)$, after delivery in 22 patients $(63 \%)$, at autopsy in one patient (3\%), and unknown in one patient (3\%). The symptoms mostly suggestive of pheochromocytoma were paroxysmal hypertension in $27(77 \%)$ of the 35 patients. The clinical symptoms generally appeared in the third trimester in 23 patients $(66 \%)$. Pheochromocytoma is a rare disease, but a high index of clinical suspicion must be kept in mind and pheochromocytoma must be listed in the differential diagnosis of hypertension associated with pregnancy. Recently, abdominal ultrasound study and MR imaging can be used to localize the tumor during the antepartum period. Once the diagnosis is confirmed, alpha-blockade is essential and betablockade may be required. In the first and second trimesters, tumor resection has a good fetal outcome; in later pregnancy, delivery by elective caesarean section followed by tumor resection is recommended.

\section{Acknowledgements}

This study was supported in part by a grant from the Ministry of Health and Welfare, Disorders of Adrenal Hormones Research Committee, Japan.

\section{References}

1. Peelen JW, DeGroat A (1955) Pheochromocytoma complicated by pregnancy. Am J Obstet Gynecol 69: 1054-1061.

2. Harper MA, Murnaghan GA, Kennedy L, Hadden DR, Atkinson AB (1989) Phaeochromocytoma in pregnancy: five cases and a review of the literature. Brit J Obstet Gynaecol 96: 594-606.

3. Bennett M, Mather G (1959) Phaeochromocytoma in pregnancy. Lancet 1: 811-812.

4. Lamming GD, Symonds EM, Rubin PC (1990)
Phaeochromocytoma in pregnancy: still a cause of maternal death. Clin Exp Hyper - In Pregnancy B9: $57-68$.

5. Brann WC, Puryear WG, Landes RR (1956) Pheochromocytoma in pregnancy. J Urol 76: 323-329.

6. Hendee AE, Martin RD, Waters WC (1969) Hypertension in pregnancy: toxemia or pheochromocytoma? Am J Obstet Gynecol 105: 64-72.

7. Sprague AD, Thelin TJ, Dilts PV (1972) Pheochromocytoma associated with pregnancy. Obstet 
Gynecol 39: 887-891.

8. Venuto R, Burstein P, Schneider R (1984) Pheochromocytoma: antepartum diagnosis and management with tumor resection in the puerperium. Am J Obstet Gynecol 150: 431-432.

9. Schenker JG, Granat M (1982) Phaeochromocytoma and pregnancy: an updated appraisal. Aust NZ J Obstet Gynaecol 22: 1-10.

10. Oishi S, Umeda T, Sagara K, Sato T, Soejima H, Ikegami K (1978) A case of multiple ectopic pheochromocytomas occurring in the urinary bladder and the para-aortic region. Jap J Clin Oncol 8: 55-61.

11. Oishi S, Shimada T, Inoue J, Sato T (1985) A case report of pheochromocytoma associated pregnancy and review of the Japanese literature. Jpn J Intern Med 74: 467-472 (In Japanese).

12. Hume DM (1960) Pheochromocytoma in the adult and in the child. Am J Surg 99: 458-496.

13. Barzel US, Bar-Ilan Z, Rumney G, Lazebnik Y, Eckerling B, DeVries A (1964) Pheochromocytoma and pregnancy: simultaneous delivery by cesarean section and resection of tumor. Am J Obstet Gynecol 89: 519-527.

14. Combs CA, Easterling TR, Schmucker BC,
Benedetti TJ (1989) Hemodynamic observations during paroxysmal hypertension in a pregnancy with pheochromocytoma. Obstet Gynecol 74: 439441.

15. Burgess III GE (1979) Alpha blockade and surgical intervention of pheochromocytoma in pregnancy. Obstet Gynecol 53: 266-270.

16. Falterman CJ, Kreisberg R (1982) Pheochromocytoma: Clinical diagnosis and management. South Med J 75: 321-328.

17. Fink IJ, Reinig JW, Dwyer AJ, Doppman JL, Linehan WM, Keiser HR (1985) MR imaging of pheochromocytomas. J Comput Assist Tomogr 9: 454-458.

18. Greenberg M, Moaward AH, Wieties BM, Goldberg LI, Kaplan EI (1986) Extra-adrenal pheochromocytoma: detection during pregnancy using MR imaging. Radiol 161: 475-476.

19. Oliver MD, Brownjohn AM, Vinall PS (1990) Medical management of phaeochromocytoma in pregnancy. Aust NZ J Obstet Gynaecol 30: 268-271.

20. Fudge TL, McKinnon WMP, Geary WL (1980) Current surgical management of pheochromocytoma during pregnancy. Arch Surg 115: 1244-1225. 